\title{
A MIXED-SIGNAL SENSOR INTERFACE MICROINSTRUMENT
}

\author{
Keith L. Kraver ${ }^{a}$, Matthew R. Guthaus ${ }^{a}$, Timothy D. Strong ${ }^{a}$, \\ Peter L. Bird ${ }^{\mathrm{a}}$, Geun Sig Cha ${ }^{\mathrm{b}}$, Wolfgang Höld ${ }^{\mathrm{c}}$, Richard B. Brown ${ }^{\mathrm{a}}$ \\ ${ }^{a}$ Department of Electrical Engineering and Computer Science, The University of Michigan, Ann Arbor, MI 48109 \\ ${ }^{b}$ Chemical Sensor Research Group, Department of Chemistry, Kwangwoon University, Seoul, Korea \\ ${ }^{c}$ Personal Systems Division, National Semiconductor Corporation, D-82256 Fürstenfeldbruck, Germany
}

\begin{abstract}
A single-chip implementation of a microinstrumentation system is presented. The chip incorporates voltage, current, and capacitive sensor interfaces; a temperature sensor; a 10-channel, 12-bit analog-to-digital converter; and an 8-bit microcontroller with a 16-bit hardware multiplier and a 40-bit accumulator. Standard serial and parallel interfaces facilitate digital communication with a host system. Fabricated in a standard $0.35 \mu \mathrm{m}$ digital CMOS process, the die occupies $3.8 \mathrm{~mm} \times 4.1 \mathrm{~mm}$, operates from a nominal supply voltage of $3 \mathrm{~V}$, and draws $16 \mathrm{~mA}$ when fully powered ( $850 \mu \mathrm{A}$ standby current). For testing purposes the chip is packaged in a 132-pin ceramic PGA.
\end{abstract}

\section{INTRODUCTION}

Sensor interfaces have been identified as critical to the development of the MEMS field [1]. Multi-chip sensor/interface-circuit solutions have been developed [2], and interface electronics have been integrated with sensors [3]. This paper presents a single-chip microinstrument: a mixed-signal microcontroller with a full analog front end--including voltage, current, and capacilive sensor interfaces; signal amplification; and analog to digital conversion circuitry. The only sensor integrated on this chip is for temperature, but other sensors and actuators could be included. For many applications, though, in which the MEMS devices cannot be made with CMOS process steps or in which sensor lifetime is limited, the most efficient system partitioning allocates all or most of the electronics on a microinstrument chip separate from the sensor.

The mixed-signal microinstrument, named the MS-8, is optimized, from instruction set definition to analog functionality, to economically support embedded sensor applications. See the block diagram in Figure 1 [4]. A minimal version of the microinstrument can be implemented with as few as eight pins. The instruction set provides excellent code density and supports the ANSI C programming language. To aid code debugging, the MS-8 includes hardware support for a single breakpoint and trace event, and a development system interface provides instructions for halting the processor, single stepping through code, and reading and writing system registers. Single cycle interrupt response and direct-memory access (DMA) capabilities facilitate usage of the MS-8 in timecritical applications, and a $16 \times 16$ hardware multiply, 40-bit accumulate block provides significant signal processing capability for a small microcontroller. To minimize power dissipation, data paths were limited to 8-bit widths, and the chip was designed to operate on a 3-V supply, in a $0.35-\mu \mathrm{m}$ CMOS process. In addition, a clock manager provides programmability of the clock frequency for the digital and analog circuits, and each analog block can be individually powered down under software control.

\section{DIGITAL CORE AND PERIPHERALS}

The right side of Figure 1 depicts the digital portion of the MS-8, which includes the processor core, peripherals, and mem-

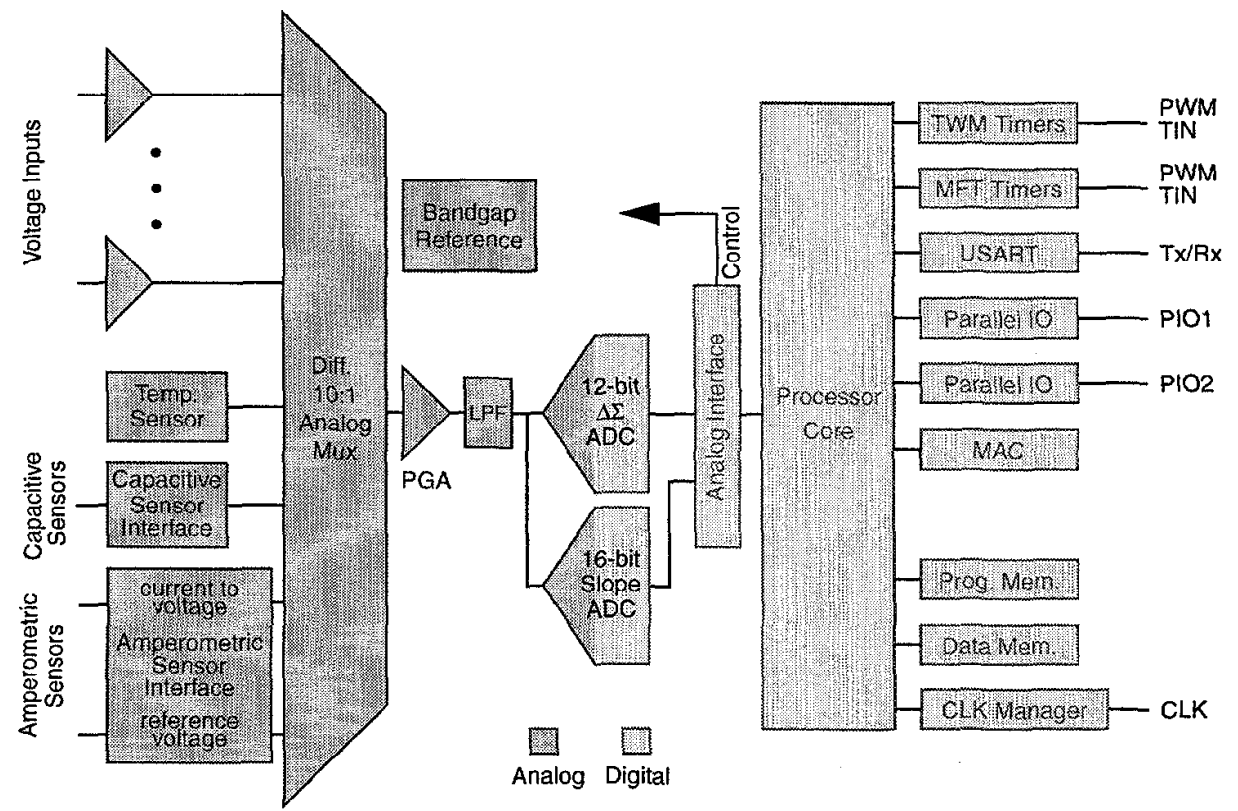

Figure 1. MS-8 block diagram. The analog (dark shade) and digital (light shade) sections interface through the analog interface block, treated as a peripheral by the processor core. 
ory [5]. Communication to a host system is facilitated through two parallel input/output units (PIO) and the universal synchronous/ asynchronous receiver/transmitter (USART). Standard timing functions are provided by the watchdog timer (TWM) and the multifunction timer (MFT). The multiply-accumulate unit (MAC) supports 16-bit multiplication and 40-bit accumulation, permitting on-chip signal processing. To reduce power, the clock manager can divide the system clock, select a slow clock, or selectively halt the clock to the different sections of the chip until a programmed event occurs. Program memory in this prototype version of the MS-8 consists of a 512 B boot ROM and a 4 KB RAM; a 512 B RAM provides data storage. For application versatility, both RAMs are configurable as either instruction or data storage. In addition, offchip memories can occupy part of the 20-bit address space. Finally, the analog interface block (AIB) contains control registers for configuring the analog circuits and data registers for buffering samples from the analog to digital converters.

\section{ANALOG CORE}

The analog front end (AFE), outlined in the left half of Figure 1, includes a multiplexor which selects from an array of buffered voltage inputs (for potentiometric chemical sensors), the temperature sensor, a capacitive sensor interface (for pressure and acceleration sensors), and an amperometric chemical sensor interface. Signal conditioning provided by the programmable gain amplifier makes the interface generic and increases the dynamic range of the system. A passive low-pass filter and a 12-bit analog to digital converter (ADC) complete the AFE. The remainder of this section summarizes the analog functionality.

The buffered voltage inputs provide a high impedance interface for potentiometric sensors or any other voltage signals in the range from 0 to $3.0 \mathrm{~V}$ (single-ended). Potentiometric chemical sensors have output impedances on the order of $100 \mathrm{M} \Omega$, which require readout circuitry with high input impcdance. The CMOS rail-to-rail opamp, described later, configured as a voltage follower, provides the necessary high input impedance huffer. Buffers not in use can be powered down selectively.

The temperature sensor, derived from the bandgap reference, is designed to provide a linear conversion of temperature to voltage in a range exceeding -40 to $85^{\circ} \mathrm{C}$ according to

$$
V(T)=-B \frac{R_{2}}{R_{1}} \ln (A) \frac{K T}{q}+V_{r e f}\left(1+\frac{R_{2}}{R_{r e f}}\right)
$$

where $B$ and $A$ are temperature-independent constants, the $R$ s are carefully ratioed resistors, and $V_{\text {ref }}$ is a temperature-independent voltage generated by the on-chip bandgap reference. Temperature range and sensitivity are competing characteristics set by the gain of the programmable gain amplifier, discussed later.

The capacitive interface circuit converts a capacitance to a voltage. Capacitive pressure sensors and accelerometers operate by deforming a capacitor plate from the steady state position in response to an applied force [2]. In the readout circuit shown in Figure 2, a switched-capacitor charge integrator compares the resulting sensor capacitance to a rcference capacitor according to

$$
V_{\mathrm{uut}}=V_{A} \frac{\left(C_{s}-C_{r e f}\right)}{C_{f}}+V_{r e f},
$$

where $C_{s}$ and $C_{r e f}$ are the sensor and reference capacitors, respectively; $V_{A}$ is the amplitude of the clock, $\phi$; and $V_{r e f}$ is the on-chip reference voltage. Through program control, the user can select an

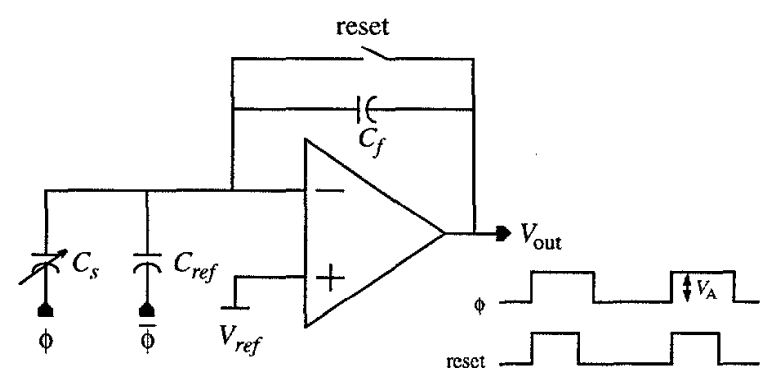

Figure 2. Simplified circuit diagram of the capacitive readout circuit.

on-chip programmable capacitor bank or an off-chip reference capacitor.

Amperometric sensors are frequently employed to detect dissolved gases or uncharged molecules in liquid. As shown in Figure 3 , these sensors typically include three terminals: counter, reference, and working. The opamps connected to the counter and reference terminals create a potentiostat circuit whereby feedback regulates the applied voltage across the counter and working terminals independent of the current in the cell. In this version of the MS-8, a simple ramp generator, shown functionally in Figure 3, generates the applied voltage. Current sensing occurs at the working electrode with a simple current follower amplifier which outputs a voltage proportional to the cell output current. To facilitate varying sensors and current magnitude, $R_{f}$ is programmable through the AIB or supplied off-chip.

The 10:1 differential analog mux consists of two 10:1 singleended muxes. Each of the previously mentioned mux inputs feeds a channel of both single-ended muxes. With this connection scheme, the mux provides maximum versatility in selecting inputs. The AIB contains a register and logic for configuring the mux under program control.

In this implementation of the MS-8, the programmable gain amplifier (PGA) is designed after the standard three-opamp, twostage instrumentation amplifier. (See Figure 4.) The first stage provides large input impedance, a differential input, and programmable gains of 1 to 61 in steps of $10 \mathrm{~V} / \mathrm{V}$. Stage two of the amplifier provides low output impedance, differential to signal-ended conversion, and level shifting. The level shifting maintains the DC level of the signal at mid-rail to avoid clipping. Use of the rail-torail opamp (described later) in both stages provides the PGA with a wide dynamic range. At the output of the PGA, a simple low-pass filter reduces amplifier distortion and aliasing (resulting from the sampling process). An AIB control register stores the gain settings.

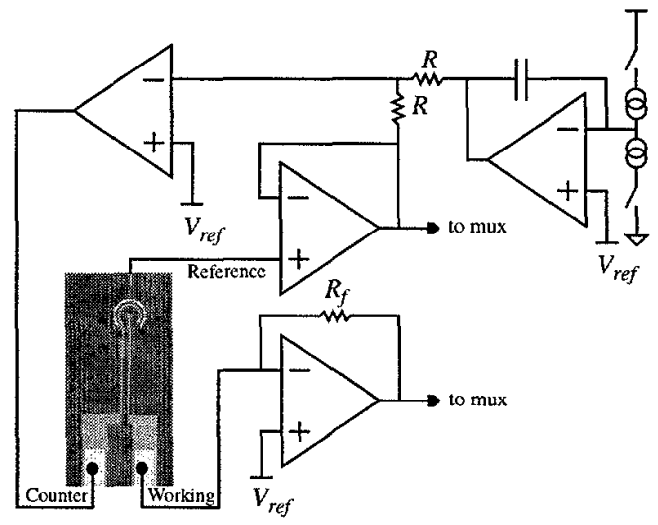

Figure 3. Simplified diagram of the amperometric sensor interface circuit and off-chip sensor. 


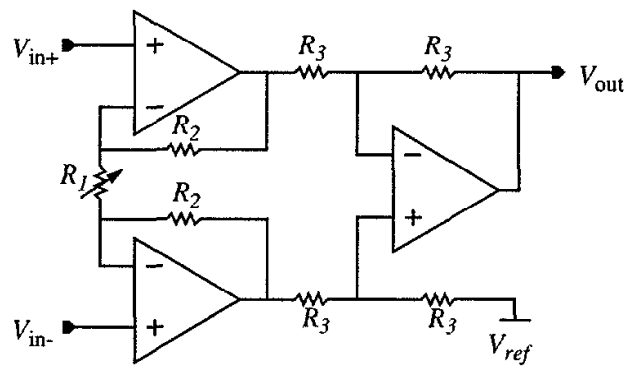

Figure 4. Programmable instrumentation amplifier. Gain is set by an on-chip programmable resistor $\left(R_{1}\right)$.

The bandgap reference supplies bias current and a voltage reference for the analog circuitry. An opamp in a negative-feedback configuration provides voltage gain to boost the reference voltage to mid-rail and buffering to drive the reference to the $\Delta \Sigma$ $A D C$, programmable gain amplifier, temperature sensor, potentiostat; and off-chip. To avoid start-up transient behaviors, the bandgap circuit is powered even in low-power mode.

Two selectable data converters produce a digital representation of the analog signal. The single-slope ADC has higher resolution than the $\triangle \Sigma$ ADC but is more susceptible to substrate noise. Because of this limitation, the single-slope converter will be useful for studying noise-reduction methods in this mixed-signal environment. The ramp voltage for the converter is generated by supplying a fixed current, derived from the bandgap reference, to an offchip capacitor.

The first-order $\Delta \Sigma$ ADC (the primary data converter) is shown in Figure 5. Under program control, the clock manager divides the system clock to generate the sampling clock and permits the user to vary the sampling frequency according to the application. In addition, the clock manager generates the four clock phases shown in the figure. This clocking scheme reduces the impact of switch-induced charge injection on the high impedance nodes.

A 16-bit counter in the AIB filters and decimates the oversampled, single-bit ADC output. The AIB stores both the counter output and the single-bit oversampled output, allowing the processor core to provide the filtering and decimation, if desired by the user.

A custom opann, based on a topology in [6], was designed for use in the analog circuits described previously. Since in some cases the opamp drives the signal off-chip (e.g. the reference voltage and test points), the opamp is designed to handle $200 \mathrm{pF}$ loads. To increase dynamic range in this low-voltage environment, the opamp processes rail-to-rail input signals and drives rail-to-rail output signals. A circuit that maintains constant transconductance, $g_{m}$, over the input common mode ranges reduces distortion.

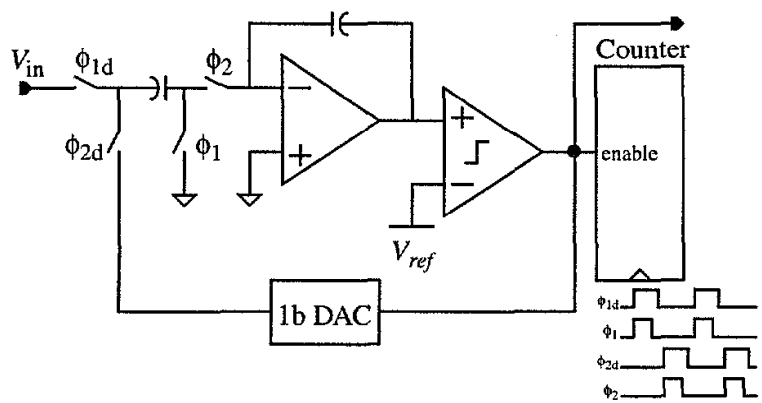

Figure 5. Simplified diagram of first-order $\Delta \Sigma$ modulator. Filtering is provided by the counter or with software and the MAC peripheral.

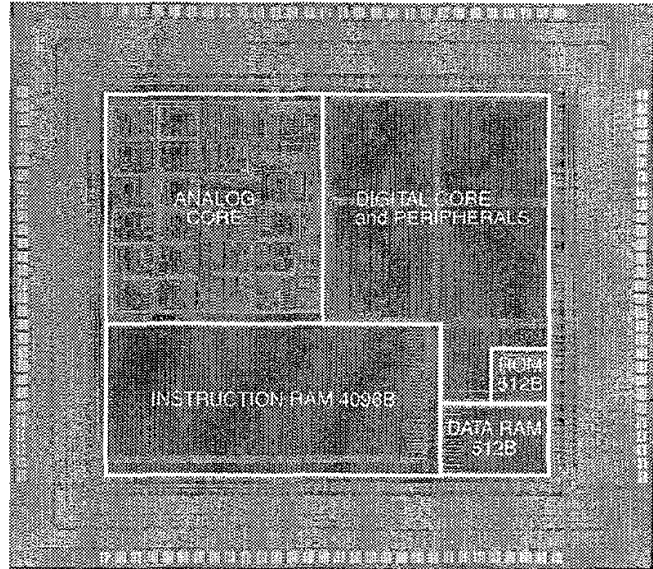

Figure 6. MS-8 die photomicrograph.

Finally, the transistors in the input differential pair and output stage operate in weak inversion to reduce input referred noise and offset, and to maximize $g_{m}$ for a given current.

\section{PHYSICAL DESIGN}

Figure 6 is a die photomicrograph of the MS-8. The major components visible are the RAMs, ROM, analog circuitry, and the microcontroller core. The die is approximately $3.8 \mathrm{~mm} \mathrm{x} 4.1 \mathrm{~mm}$. Nearly one-quarter of the core area, $1.39 \mathrm{~mm} \times 1.42 \mathrm{~mm}$, is dedicated to the analog circuits. RAM and ROM memory cells occupy a little less than half of the core.

The memory drivers are placed as far from the analog circuits as possible to reduce substrate coupling effects. In addition, the analog core has ample substrate contacts surrounding the sensitive circuitry and dedicated $V_{d d}$ and ground connections to further combat switching noise. Finally, enforcing metal-to-metal spacings greater than minimum reduces crosstalk between signal lines (at the expense of area).

\section{EXPERIMENTAL RESULTS}

The MS-8 digital core has been verified to be functional at $40 \mathrm{MHz}, 3.0 \mathrm{~V}$, and $75^{\circ} \mathrm{F}$ using an HP82000 digital tester. While simulations predict static current draw around $3.3 \mathrm{~mA}$ (full power) and $15 \mu \mathrm{A}$ (standby), measurements reveal $16 \mathrm{~mA}$ and $850 \mu \mathrm{A}$. Initial testing of the AFE reveals the usefulness of the device in embedded sensor applications. The USART enabled bidirectional serial communications between the MS-8 and a laptop computer running LabView (National Instruments). Specifically, programs were downloaded to the chip and digital samples from the ADC were transferred to LabView for recording and analysis. The potentiometric and capacitive sensor interfaces and the temperature sensor have been verified. In addition, the ramp generator circuit in the amperometric interface has been successfully tested, and amperometric sensors are being prepared to complete the demonstration of the MS-8 analog interfaces.

Figure 7 shows the calibration curve over seven decades of $\mathrm{KCL}$ concentration for a solid-state potentiometric sensor selective to potassium ions $\left(\mathrm{K}^{+}\right)$[7]. The inset contains the time response of the sensor; each step represents an 10-fold increase in potassium concentration. A wire from the output pin of the on-chip reference generator set the potential of the solution to $1.5 \mathrm{~V}$. The sensor has a linear response from around the minimum detectable limit of $10^{-5} \mathrm{M}$ to about $1 \mathrm{M}$, as shown in the figure. Using the 


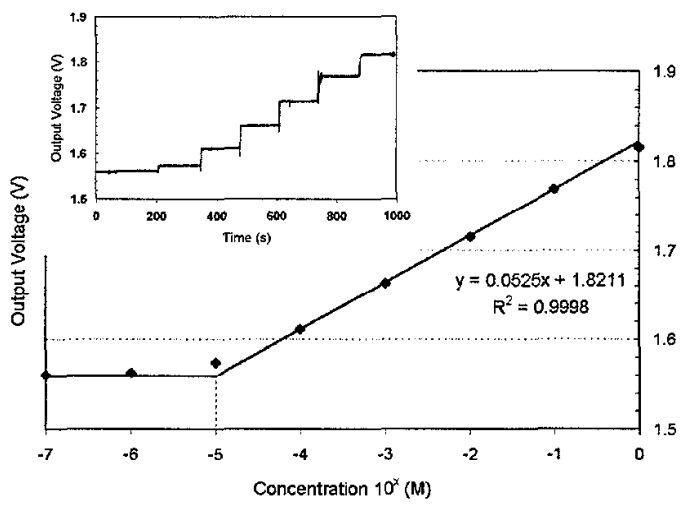

Figure 7. $\mathrm{K}^{+}$calibration curve. The background solution consists of $200 \mathrm{ml}$ of $0.05 \mathrm{M}$ Trizma Base (Sigma, St. Louis, MO) adjusted to $\mathrm{pH} 7.4$ with $\mathrm{H}_{2} \mathrm{SO}_{4}$. The PGA gain equals $1 \mathrm{~V} / \mathrm{V}$.

microcontroller to linearize the response with a higher-order fit would extend the detection range.

The initial test of the capacitive interface circuit was performed with an $8.2 \mathrm{pF}$ external reference capacitor and known capacitances to represent the sensor, see Figure 8. Actual capacitive-based pressure sensors will be interfaced to the MS- 8 in the future. The figure shows a linear capacitance to voltage conversion. Pressure sensors, however, demonstrate a nonlinear relationship between pressure and output capacitance, which could be linearized with the signal processing capabilities of the MS-8.

To test the temperature sensor, the MS- 8 and test-board were placed in an oven. The temperature was ramped slowly from room temperature, $22^{\circ} \mathrm{C}$, to $80^{\circ} \mathrm{C}$, as measured by a mercury thermometer. At each temperature, the chip was allowed to reach equilibrium with the oven environment before a digital voltage reading was recorded. As expected, Figure 9 shows the temperature sensor output voltage has a linear relationship to temperature.

\section{CONCLUSIONS}

The MS-8 described here is the first implementation of an on-going project that coordinates efforts among analog designers, compiler writers, and low-power digital designers to develop a low-power, low-cost, and multipurpose sensor interface and data acquisition system. This single-chip microinstrument contains a programmable analog front end capable of interfacing to a variety of sensors. The integrated microcontroller and peripherals support digital filtering and compensation of sensor outputs, timing control

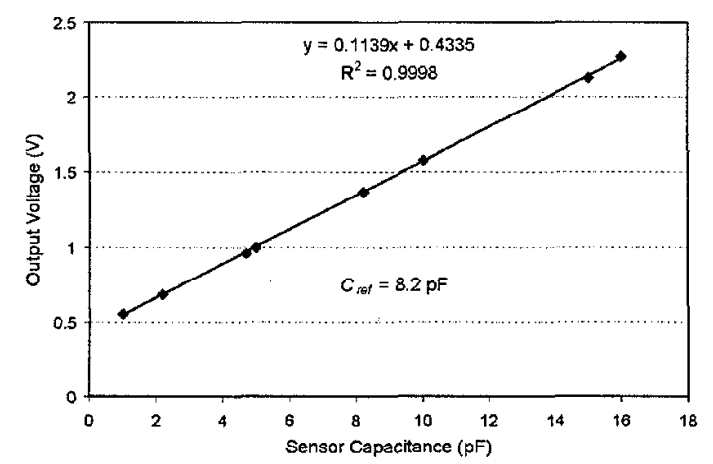

Figure 8. Capacitive sensor readout circuit test. The PGA gain is set to $1 \mathrm{~V} / \mathrm{V}$.

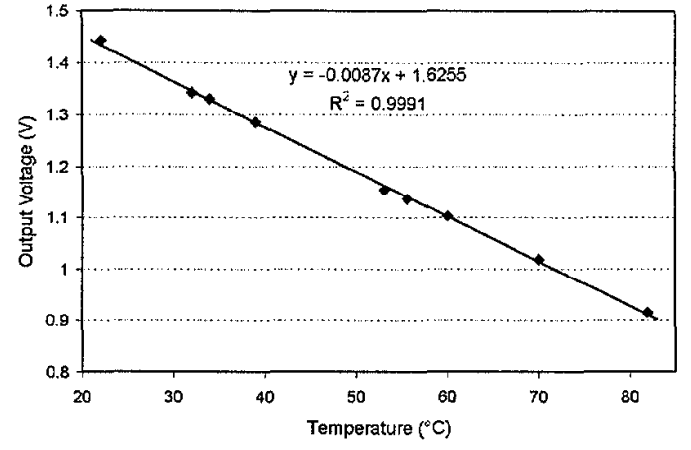

Figure 9. Temperature sensor response from 25 to $80^{\circ} \mathrm{C}$ with $P G A$ gain set to $11 \mathrm{~V} / \mathrm{V}$.

for sampling multiple sensors, and communication with a host system. The usefulness of the MS- 8 in embedded sensor applications is evidenced by the initial results presented. More testing with pressure and chemical sensors is planned to further demonstrate the capabilities of the MS-8.

\section{ACKNOWLEDGMENTS}

The authors wish to thank E. Dietz, H. Verhoeven, and D. Vieira of National Semiconductor for discussions concerning the design of the opamp and review of the analog layout; $M$. Lortz and M. Embacher from National Semiconductor for support with the physical design; and A. Drake from the University of Michigan for assistance with digital testing.

\section{REFERENCES}

1. K. D. Wise, Ed., Proc. IEEE Special Issue on Integrated Sensors, Microactuators, and Microsystems (MEMS), August 1998.

2. A. V. Chavan, A. Mason, U. Kang, and K. D. Wise, "Programmable Mixed-Voltage Sensor Readout Circuit and Bus Interface with Built-In Self-Test," IEEE ISSCC Digest of Technical Papers, pp. 136-137, February 1999.

3. H. D. Goldberg, The Batch Fabrication of Integrated Chemical Sensor Arrays, Ph.D. Dissertation, The University of Michigan, 1993.

4. P. L. Bird, R. B. Brown, T. N. Mudge, L. Hong, and B. Khailany, "MS-8 Design Specification," Technical Report, The University of Michigan, January 1999.

5. R. B. Brown, P. L. Bird, M. R. Guthaus, K. L. Kraver, and B. Khailany, "MS-8 Microarchitecture," Technical Report, The University of Michigan, May 1999.

6. R. Hogervorst and J. H. Huijsing, Design of Low-Voltage, Low-Power Operational Amplifier Cells. Klewer, Boston, 1996.

7. H. J. Yoon, J. H. Shin, H. Nam, G. S. Cha, T. D. Strong, and R. B. Brown, "Solid-State Ion Sensors with a Junction-Free Solvent-Processible Polymer Membrane-Based Reference Electrode for Blood Analysis," 10th International Conference on Solid-State Sensors and Actuators, Transducers '99, Sendai, Japan, pp. 652655, June 7-10, 1999. 